\title{
There is variability in the attainment of developmental milestones in the CDKL5 disorder
}

\author{
Stephanie Fehr ${ }^{1}$, Helen Leonard', Gladys Ho ${ }^{3}$, Simon Williams ${ }^{5}$, Nick de Klerk' ${ }^{1}$ David Forbes ${ }^{6}$, \\ John Christodoulou ${ }^{3,4}$ and Jenny Downs ${ }^{1,2^{*}}$
}

\begin{abstract}
Background: Individuals with the CDKL5 disorder have been described as having severely impaired development. A few individuals have been reported having attained more milestones including walking and running. Our aim was to investigate variation in attainment of developmental milestones and associations with underlying genotype.

Methods: Data was sourced from the International CDKL5 Disorder Database, and individuals were included if they had a pathogenic or probably pathogenic CDKL5 mutation and information on early development. Kaplan-Meier time-to-event analyses investigated the occurrence of developmental milestones. Mutations were grouped by their structural/functional consequence, and Cox regression was used to investigate the relationship between genotype and milestone attainment.

Results: The study included 109 females and 18 males. By 5 years of age, only 75\% of the females had attained independent sitting and 25\% independent walking whilst a quarter of the males could sit independently by 1 year 3 months. Only one boy could walk independently. No clear relationship between mutation group and milestone attainment was present, although females with a late truncating mutation attained the most milestones.

Conclusion: Attainment of developmental milestones is severely impaired in the CDKL5 disorder, with the majority who did attain skills attaining them at a late age. It appears as though males are more severely impaired than the females. Larger studies are needed to further investigate the role of genotype on clinical variability.
\end{abstract}

Keywords: CDKL5 disorder, Developmental disabilities, Epileptic encephalopathy, Early infantile epileptic encephalopathy

\section{Background}

The majority of research on individuals with mutations in the cyclin-dependent kinase-like 5 (CDKL5) gene has been limited to case studies and small case series $(n=20)$ [1-3]. These studies have predominantly focused on individuals with the early-onset seizure variant of Rett syndrome (RTT). However, using information collected through the International Rett Syndrome Phenotype Database (InterRett) [4], we found that only a small proportion of individuals with a CDKL5 mutation met the clinical criteria for the early-onset seizure variant of RTT, which specifically requires a period of developmental

\footnotetext{
*Correspondence: Jenny.Downs@telethonkids.org.au

${ }^{1}$ Telethon Kids Institute, University of Western Australia, Perth, Western

Australia, Australia

${ }^{2}$ School of Physiotherapy and Exercise Science, Curtin University, Perth,

Western Australia, Australia

Full list of author information is available at the end of the article
}

regression [5]. We therefore suggested that the term 'CDKL5 disorder' was a more appropriate terminology for individuals with a mutation in the CDKL5 gene.

The literature suggests that individuals with the CDKL5 disorder rarely experience normal development and that the majority have refractory epilepsy with onset within the first few months of life and severe global developmental delay [6-8]. There has however been a small proportion reported with the ability to walk and even to run [9-12], suggesting that the clinical severity may be more variable than originally thought. Variability in clinical presentation is also seen in RTT, which is mostly associated with mutations in the methyl-CpG binding domain-2 gene (MECP2) [5]. This clinical variability has resulted in 'variant' types being described and studies focusing on investigating the relationship between clinical presentation and specific mutations [13-15]. For caregivers and clinicians, it is 
important that variability in the CDKL5 disorder be further examined so that appropriate prognostic information can be provided. Further investigation is needed to develop a better clinical overview of the development and attainment of milestones in this disorder. Our aim was to describe the attainment of gross motor, hand function, communication and other developmental milestones in individuals with the CDKL5 disorder. We also investigated the relationship between the timing of these events and genotype.

\section{Methods}

Information on individuals with the CDKL5 disorder was obtained from the newly developed International CDKL5 Disorder Database, which was established in September 2012. Data is collected from caregivers in the form of online or paper-based questionnaires. Caregivers were asked to refer to their child's infant health record, diaries or available medical records when completing the questionnaire. Cases were included in this study if the individuals CDKL5 mutation was considered to be pathogenic or probably pathogenic, and the caregiver had completed the questionnaire section on early development. Due to the heterogeneity of mutations within our sample, each individual's mutation was grouped according to its predicted structural/functional consequence [16]. These groups were a) mutations causing no functional protein which included mutations resulting in a loss of the functional components in the catalytic domain (portion of protein responsible for kinase activity) before amino acid (aa) 172 and all full gene deletions, b) missense/in-frame mutations within the catalytic domain which included any missense mutations within the protein kinase active region or in-frame mutations (such as a deletion resulting in loss of some of the kinase region but subsequent protein is intact), c) truncating mutations located from aa 172 to aa 781 inclusive which includes any mutations resulting in a truncation such as nonsense or frameshift mutations resulting in maintaining kinase activity but loss of c-terminal region and d) truncating mutations occurring after aa 781 and therefore maintaining kinase activity and majority of the c-terminal region (Figure 1).

To investigate early development, we included information on the age of attainment of the following major developmental milestones: sitting, standing and walking independently; raking grasp and pincer grip; and babble and use of single words. The timing of attainment of these milestones was investigated using Kaplan-Meier time-toevent analysis. This allows an appropriate estimate of the proportion of individuals who will attain a skill by a particular age whilst taking into account those who are still yet to attain the skill. To investigate the relationship between mutation type and the attainment of these particular milestones, separate time-to-event analyses by mutation group were undertaken and the median age and proportion that attained each skill determined. Cox regression was used to investigate the relationship between each skill and mutation group.

Information on the attainment of additional milestones, for which we did not collect an age of attainment, was also included. For these skills, individuals were grouped according to their current age at time questionnaire completion (younger than 1.5 years; 1.5 to $<7$ years; 7 to $<13$ years; and 13 years and older) and we determined the proportion of each age group who had attained the milestone of interest. These age groups were chosen based on the expected developmental level of the children. All the milestones included in our study (excluding the use of phrases) would have been achieved by 1.5 years of age in a normally developing child. We therefore grouped those aged 1.5 years and under as it was likely that these children were yet to attain many of these skills. This allowed us to account for young individuals who had not yet had the opportunity to attain these skills.
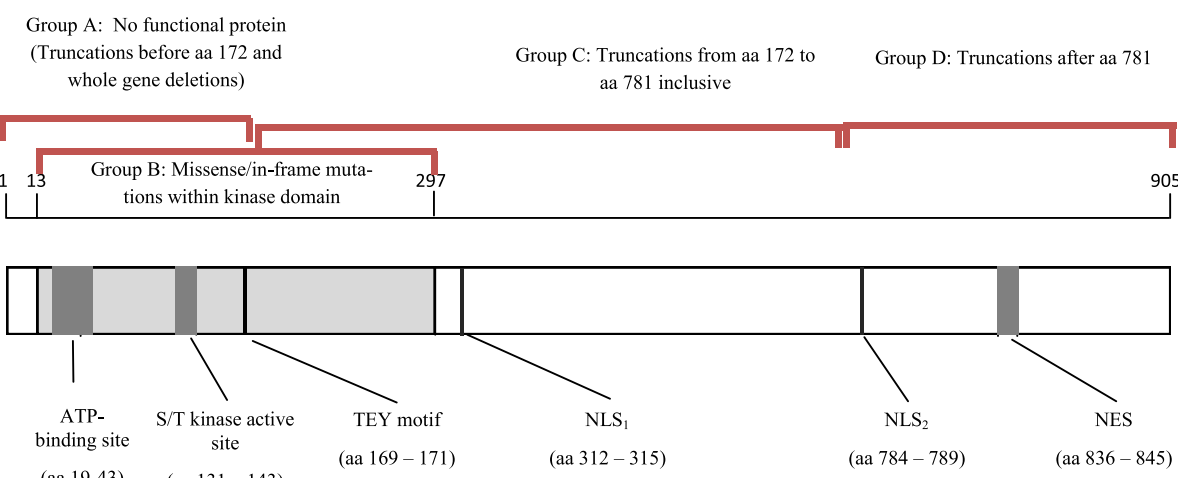

Figure 1 Schematic representation of the CDKL5 protein and our mutational grouping. Legend: The catalytic region is shown in light grey and the C-terminal region in white. Modified from 'What We Know and Would Like to Know about CDKL5 and its Involvement in Epileptic Encephalopathy' [17]. TEY motif Thr-Glu-Tyr motif, NLS, and NLS, nuclear localisation signals 1 and 2, NES nuclear export signal. 
Approval for this study was provided by the University of Western Australia Human Research Ethics Committee, Perth, Western Australia, Australia (RA/4/1/5024).

\section{Results}

As of May 2014, there were 127 individuals for whom information on early development had been provided. Of these, 109 (86\%) were female (median age 6.1 years, range 3 months to 29 years) and 18 (14\%) were male (median age 4.6 years, range 10 months to 22.7 years). The distribution of mutations within our sample is shown in Table 1. We have also included information on our determination of whether missense mutations were likely pathogenic (Table 2).

\section{Time-to-event analysis of attainment of developmental milestones}

Information on the age of attaining independent sitting was available for all but four females, of whom 59 (56\%) had attained independent sitting. The median age at attainment of independent sitting was 3 years (range 6 months to 5 years) with almost three quarters achieving unaided sitting by 5 years of age (Figure 2). Independent standing was attained by $26 \%$ of females $(n=27 / 105)$, and a quarter was able to stand independently by 5 years (range 10 months to 6 years 11 months). Independent walking was attained by $22 \%$ of females $(n=24 / 109)$, and time-to-event analysis showed that a quarter had attained independent walking by 4 and a half years (range 1 year 6 months to 6 years 5 months). For the 18 boys in our study, information on the age of attaining sitting was known for all but one, of whom six could sit independently with a quarter attaining independent sitting by 1 year 3 months (range 8 months to 3 years) (Figure 2). Two boys were able to stand independently by 1 year 10 months, and one boy was able to walk independently at 1 year 11 months.

The age that a raking grasp was attained was known for 92 females of whom 45 (49\%) had attained a raking grasp at a median age of 5 years (range 5 months to 12 years) (Figure 2). A pincer grasp was attained by $13 \%$ of females $(n=14 / 106)$ of whom $10 \%$ had attained a pincer grip by 3 years (range 1 to 12 years). For the males, information was available for all but one on the age of attaining a raking grasp. Ten percent of the males attained a raking grasp by 1 year 10 months and one male attained a pincer grasp at 2 years of age (Figure 2).

Information on the age that babbling was attained was available for 97 females of whom 43 (44\%) had attained babble. A quarter of whom achieved babble by 1 year of age (range 3 months to 6 years) and just under a half by 6 years (Figure 2). Single words were attained by $16 \%(n=17 / 105)$, and $10 \%$ would attained single words by 1 year 6 months and just under a quarter by 7 years of age (range 9 months to 7 years). Of the males in our study, information on age of attaining babble was available for all but three $(n=15)$ of whom five attained the ability to babble. A quarter attained babble by 7 months of age (range 6 months to 1 year 8 months) (Figure 2). There was one boy who spoke single words at 3 years of age.

The proportion of females and males who attained other gross motor, fine motor and communication skills not included in the time-to-event analysis is shown in Table 3.

\section{Relationship between genotype and attainment of developmental milestones for females}

A higher proportion of females with a truncating mutation after aa 781 attained gross motor, hand function and communication milestones than the other groups (Table 4). Although there was a visible difference between the groups shown in Figure 3, there were no clear relationships, although females with a truncating mutation after aa 781 were more likely to have attained independent sitting than the those with no functional protein (HR: 2.4, $p$ value 0.026, 95\% CI 1.11-5.36).

\section{Discussion}

The acquisition of developmental milestones was severely impaired in our sample of individuals with the CDKL5 disorder. This was especially true for males, with nearly half not achieving any of the major gross motor, communication or hand function milestones investigated. There was variability, with a few girls eventually attaining most if not all of the milestones we investigated. If milestones were attained, they were significantly delayed. Despite small numbers, it appears as though mutation type may play some role in this variation, with girls with a truncating mutation after aa 781 attaining more milestones than those who had a mutation resulting in no functional CDKL5 protein.

Our study has shown that there is variability in the attainment of developmental milestones within the CDKL5 disorder. The majority of females did achieve some milestones, most frequently involving gross motor skills. For most, these milestones were attained at an older age than expected for the general population [30]. Using time-toevent analysis, we found that $10 \%$ of females would attain independent sitting by 10 months, by 1 year 2 months a quarter attained independent sitting and by 5 years nearly three quarters had attained independent sitting. In our entire female population, only $22 \%$ attained independent walking compared to $31 \%$ of our 7 - to 13 -year group. Timeto-event analysis indicated that $25 \%$ of our group attained this skill by 4 and a half years of age. This delay in attainment of milestones occurred across developmental areas and may explain why other studies have found females with the CDKL5 disorder to attain less developmental milestones 
Table 1 Distribution of CDKL5 mutations within our study group

\begin{tabular}{|c|c|c|c|c|c|c|}
\hline Mutation group & Nucleotide change (cDNA) & Protein change & Exon/intron & $\begin{array}{l}\text { Individuals } \\
\text { (gender) }\end{array}$ & $\begin{array}{l}\text { Reported } \\
\text { mutation }\end{array}$ & $\begin{array}{l}\text { Reported } \\
\text { individual }\end{array}$ \\
\hline No functional protein & c.-253-?_-163+?del & p.(0) & 1 & $2(F)$ & Yes & Yes [4] \\
\hline No functional protein & c.-253-?_-163+?del & p.(0?) & 1 & $1(\mathrm{~F})$ & Yes & Yes [4] \\
\hline No functional protein & c.-253-?_99+?del & p.(0) & $1-3$ & $1(F)$ & Yes & Yes [4] \\
\hline No functional protein & $c .64+2 \mathrm{~T}>\mathrm{C}$ & p.(0) & 2 & $1(\mathrm{~F})$ & Yes & No \\
\hline No functional protein & $\mathrm{c} .64+1 \mathrm{G}>\mathrm{A}$ & p.(0) & 2 & $1(F)$ & Yes & Yes [4] \\
\hline No functional protein & c.-162-?_99+?del & p.(0) & $2-3$ & $1(\mathrm{~F})$ & Yes & Yes [4] \\
\hline No functional protein & C.-162-?_99+? & p.(0) & $2-3$ & $1(F)$ & No & No \\
\hline No functional protein & c.-253-?_*1085del & p.(0) & $1-21+$ & $1(F)$ & No & No \\
\hline No functional protein & c.-253-?_*1085del & p.(0) & $1-21$ & $3(F)$ & No & No \\
\hline No functional protein & c.-253-?_*1085del & p.(0) & $1-21+$ & $1(F)$ & No & No \\
\hline No functional protein & c.-253-?_*1085del & p.(0) & $1-21+$ & $1(F)$ & Yes & Yes [4] \\
\hline No functional protein & c.-253-?_*1085del & p.(0) & $1-21+$ & $1(F)$ & No & No \\
\hline No functional protein & c.65-?_99+?del & p.(Ala23Asnfs*3) & 3 & $2(\mathrm{~F}$ and $\mathrm{M})$ & Yes & Yes [4] \\
\hline No functional protein & $C .99+5 G>A$ & p.Ala23Asnfs*3 & 3 & $1(F)$ & Yes & Yes $[2,4]$ \\
\hline No functional protein & c. $100-?{ }_{-}^{*} 1085 \mathrm{del}$ & p.(?) & $4-21+$ & $1(F)$ & No & No \\
\hline No functional protein & c.146-1G > A & p.(Asn50Tyrfs*15) & 5 & $1(\mathrm{M})$ & No & No \\
\hline No functional protein & c.146-6 T > G & p.(Glu49Valfs*2) & 5 & $1(F)$ & No & No \\
\hline No functional protein & c.146-?_282+?del & p.(Asn50Tyrfs*15) & 5 & $1(F)$ & Yes & Yes [4] \\
\hline No functional protein & c.163_166delGAAA & p.Glu55Argfs*20 & 5 & $1(\mathrm{~F})$ & Yes & No \\
\hline No functional protein & c. $175 \mathrm{C}>\mathrm{T}$ & p.Arg59* & 5 & 5 (4 and $1 \mathrm{M})$ & Yes & Yes $[4,18,19]$ \\
\hline No functional protein & c.183delT & p.Met63Cysfs*13 & 5 & 2 (F, twins) & Yes & Yes [4] \\
\hline No functional protein & $c .220 G>T$ & p.Glu74* & 5 & $1(F)$ & No & No \\
\hline No functional protein & c. 282 + 3_282 + 6delAAGT & p.(Asn50Tyrfs*15) & 5 & $2(F)$ & Yes & Yes [4] \\
\hline No functional protein & c.283-3_290del & p.(Asn95llefs*2) & 5 & 2 (F, sisters) & Yes & Yes $[2,4]$ \\
\hline No functional protein & c.339ins1 & p.? & 6 & $1(F)$ & No & No \\
\hline No functional protein & c.351 T > A & p.Tyr117* & 6 & $1(\mathrm{M})$ & No & No \\
\hline No functional protein & $c .400 C>T$ & p.Arg134* & 6 & $2(\mathrm{~F}$ and $\mathrm{M})$ & Yes & Yes $[4,20]$ \\
\hline No functional protein & c.456_457delTG & p.Cys152* & 7 & $1(F)$ & No & No \\
\hline No functional protein & c.464-2A > G & p.Gly155Alafs*43 & 7 & $1(\mathrm{M})$ & Yes & No \\
\hline No functional protein & c.506_507delAC & p.Thr168Argfs*36 & 8 & $1(F)$ & No & No \\
\hline $\begin{array}{l}\text { Missense/in-frame mutation } \\
\text { within catalytic domain }\end{array}$ & $\mathrm{c} .38 \mathrm{~T}>\mathrm{C}$ & p.Phe13Ser & 2 & $1(F)$ & Yes & Yes [4] \\
\hline $\begin{array}{l}\text { Missense/in-frame mutation } \\
\text { within catalytic domain }\end{array}$ & c.58G $>C$ & p.Gly20Arg & 2 & $1(F)$ & Yes & Yes $[4,8]$ \\
\hline $\begin{array}{l}\text { Missense/in-frame mutation } \\
\text { within catalytic domain }\end{array}$ & $\begin{array}{l}\text { c.100-9_100- } \\
\text { 3delCCCTTGCinsGCAGA }\end{array}$ & p.(Lys33dup) & 4 & $1(F)$ & No & No \\
\hline $\begin{array}{l}\text { Missense/in-frame mutation } \\
\text { within catalytic domain }\end{array}$ & C.119C > T & p.Ala40Val & 4 & $1(\mathrm{~F})$ & Yes & Yes [4] \\
\hline $\begin{array}{l}\text { Missense/in-frame mutation } \\
\text { within catalytic domain }\end{array}$ & C.191 T > C & p.Leu64Pro & 5 & $1(\mathrm{M})$ & Yes & Yes $[4,21]$ \\
\hline $\begin{array}{l}\text { Missense/in-frame mutation } \\
\text { within catalytic domain }\end{array}$ & c.214_216del & p. Ile72del & 5 & $1(F)$ & No & No \\
\hline $\begin{array}{l}\text { Missense/in-frame mutation } \\
\text { within catalytic domain }\end{array}$ & C.215 T > C & p.lle72Thr & 5 & $1(F)$ & Yes & No \\
\hline $\begin{array}{l}\text { Missense/in-frame mutation } \\
\text { within catalytic domain }\end{array}$ & c. $364 \mathrm{G}>\mathrm{A}$ & p.Ala122Thr & 6 & $1(F)$ & Yes & Yes [4] \\
\hline
\end{tabular}


Table 1 Distribution of CDKL5 mutations within our study group (Continued)

\begin{tabular}{|c|c|c|c|c|c|c|}
\hline $\begin{array}{l}\text { Missense/in-frame mutation } \\
\text { within catalytic domain }\end{array}$ & $c .377 G>A$ & p.Cys126Tyr & 6 & $1(F)$ & Yes & Yes [4] \\
\hline $\begin{array}{l}\text { Missense/in-frame mutation } \\
\text { within catalytic domain }\end{array}$ & c.395 T> G & p.Val132Gly & 6 & $1(F)$ & No & No \\
\hline $\begin{array}{l}\text { Missense/in-frame mutation } \\
\text { within catalytic domain }\end{array}$ & c. $404 A>G$ & p.(Asp135_Phe154del) & 7 & $1(\mathrm{M})$ & No & No \\
\hline $\begin{array}{l}\text { Missense/in-frame mutation } \\
\text { within catalytic domain }\end{array}$ & c. $404-3 C>A$ & p.(Asp135_Phe154del) & 7 & $1(\mathrm{~F})$ & Yes & Yes [4] \\
\hline $\begin{array}{l}\text { Missense/in-frame mutation } \\
\text { within catalytic domain }\end{array}$ & c. $428 \mathrm{~T}>\mathrm{A}$ & p. Ile143Asn & 7 & $1(\mathrm{~F})$ & No & No \\
\hline $\begin{array}{l}\text { Missense/in-frame mutation } \\
\text { within catalytic domain }\end{array}$ & c. $458 \mathrm{~A}>\mathrm{T}$ & p. Asp153Val & 7 & $1(\mathrm{~F})$ & No & No \\
\hline $\begin{array}{l}\text { Missense/in-frame mutation } \\
\text { within catalytic domain }\end{array}$ & $c .463+1 G>A$ & p.Asp135_Phe154del & 7 & $1(\mathrm{~F})$ & Yes & Yes [4] \\
\hline $\begin{array}{l}\text { Missense/in-frame mutation } \\
\text { within catalytic domain }\end{array}$ & c. $473 G>C$ & p.Arg158Pro & 8 & $1(\mathrm{M})$ & Yes & Yes [4] \\
\hline $\begin{array}{l}\text { Missense/in-frame mutation } \\
\text { within catalytic domain }\end{array}$ & c. $514 G>A$ & p.Val172lle & 8 & $1(\mathrm{M})$ & No & No \\
\hline $\begin{array}{l}\text { Missense/in-frame mutation } \\
\text { within catalytic domain }\end{array}$ & C.526 T >C & p.Trp176Arg & 8 & $1(\mathrm{~F})$ & No & No \\
\hline $\begin{array}{l}\text { Missense/in-frame mutation } \\
\text { within catalytic domain }\end{array}$ & c. $532 \mathrm{C}>\mathrm{T}$ & p.Arg178Trp & 8 & $2(F)$ & Yes & Yes [4] \\
\hline $\begin{array}{l}\text { Missense/in-frame mutation } \\
\text { within catalytic domain }\end{array}$ & C. $536 \mathrm{C}>\mathrm{T}$ & p. Ser179Phe & 8 & $1(\mathrm{~F})$ & No & No \\
\hline $\begin{array}{l}\text { Missense/in-frame mutation } \\
\text { within catalytic domain }\end{array}$ & $\mathrm{C} .577 \mathrm{G}>\mathrm{C}$ & p.Asp193His & 9 & $1(\mathrm{~F})$ & No & No \\
\hline $\begin{array}{l}\text { Missense/in-frame mutation } \\
\text { within catalytic domain }\end{array}$ & $\mathrm{C} .587 \mathrm{C}>\mathrm{T}$ & p.Ser196Leu & 9 & $5(F)$ & Yes & Yes [4] \\
\hline $\begin{array}{l}\text { Missense/in-frame mutation } \\
\text { within catalytic domain }\end{array}$ & C.595 T>C & p.Cys199Arg & 9 & $1(\mathrm{~F})$ & Yes & Yes [4] \\
\hline $\begin{array}{l}\text { Missense/in-frame mutation } \\
\text { within catalytic domain }\end{array}$ & c. $620 \mathrm{G}>\mathrm{A}$ & p.Gly207Glu & 9 & $1(\mathrm{~F})$ & Yes & Yes [4] \\
\hline $\begin{array}{l}\text { Missense/in-frame mutation } \\
\text { within catalytic domain }\end{array}$ & C. $656 \mathrm{~A}>\mathrm{C}$ & p.Gln219Pro & 9 & $1(\mathrm{~F})$ & Yes & Yes [4] \\
\hline $\begin{array}{l}\text { Missense/in-frame mutation } \\
\text { within catalytic domain }\end{array}$ & c.680 T > C & p.Leu227Pro & 9 & $1(\mathrm{~F})$ & Yes & Yes [4] \\
\hline $\begin{array}{l}\text { Missense/in-frame mutation } \\
\text { within catalytic domain }\end{array}$ & $c .872 \mathrm{G}>\mathrm{A}$ & p.Cys291Tyr & 11 & $1(\mathrm{~F})$ & Yes & No \\
\hline $\begin{array}{l}\text { Truncation occurring between } \\
\text { aa } 172 \text { and } 781\end{array}$ & c. $670 \mathrm{C}>\mathrm{T}$ & p.Gln $224^{*}$ & 9 & $1(\mathrm{M})$ & No & No \\
\hline $\begin{array}{l}\text { Truncation occurring between } \\
\text { aa } 172 \text { and } 781\end{array}$ & c.556_557delGC & p.Ala186Serfs*19 & 9 & $1(\mathrm{M})$ & No & No \\
\hline $\begin{array}{l}\text { Truncation occurring between } \\
\text { aa } 172 \text { and } 781\end{array}$ & c.745-?_977 + ?del & p.(Phe249Lysfs*16) & $10-11$ & $1(\mathrm{~F})$ & No & No \\
\hline $\begin{array}{l}\text { Truncation occurring between } \\
\text { aa } 172 \text { and } 781\end{array}$ & c.801_802delAT & p.Asn267Lysfs*5 & 11 & $1(\mathrm{~F})$ & No & No \\
\hline $\begin{array}{l}\text { Truncation occurring between } \\
\text { aa } 172 \text { and } 781\end{array}$ & c.857dupA & p.Tyr286* & 11 & $1(F)$ & Yes & Yes [4] \\
\hline $\begin{array}{l}\text { Truncation occurring between } \\
\text { aa } 172 \text { and } 781\end{array}$ & c.859_868del10 & p.Leu287Serfs*3 & 11 & $1(\mathrm{~F})$ & Yes & Yes [4] \\
\hline $\begin{array}{l}\text { Truncation occurring between } \\
\text { aa } 172 \text { and } 781\end{array}$ & c.978-?_2980+?del & p.? & $12+$ & $1(\mathrm{~F})$ & No & No \\
\hline Truncation occurring between & c. $1039 C>T$ & p.Gln347* & 12 & $1(\mathrm{~F})$ & Yes & No \\
\hline
\end{tabular}


Table 1 Distribution of CDKL5 mutations within our study group (Continued)

\begin{tabular}{|c|c|c|c|c|c|c|}
\hline $\begin{array}{l}\text { Truncation occurring between } \\
\text { aa } 172 \text { and } 781\end{array}$ & c.1371dupA & p.Leu458Thrfs*5 & 12 & $1(\mathrm{~F})$ & Yes & Yes [4] \\
\hline $\begin{array}{l}\text { Truncation occurring between } \\
\text { aa } 172 \text { and } 781\end{array}$ & c. $1375 C>T$ & p.Gln459* & 12 & $1(F)$ & Yes & Yes [4] \\
\hline $\begin{array}{l}\text { Truncation occurring between } \\
\text { aa } 172 \text { and } 781\end{array}$ & c.1446delC & p.Tyr482* & 12 & $1(F)$ & Yes & Yes [4] \\
\hline $\begin{array}{l}\text { Truncation occurring between } \\
\text { aa } 172 \text { and } 781\end{array}$ & c.1470_1471delGG & p.Ala491Thrfs*3 & 12 & $1(F)$ & Yes & Yes [4] \\
\hline $\begin{array}{l}\text { Truncation occurring between } \\
\text { aa } 172 \text { and } 781\end{array}$ & c.1499ins4 & p.? & 12 & $1(\mathrm{~F})$ & Yes & Yes [4] \\
\hline $\begin{array}{l}\text { Truncation occurring between } \\
\text { aa } 172 \text { and } 781\end{array}$ & c.1547_1554del8 & p.Tyr516Phefs*2 & 12 & $1(\mathrm{~F})$ & No & No \\
\hline $\begin{array}{l}\text { Truncation occurring between } \\
\text { aa } 172 \text { and } 781\end{array}$ & c.1581del & p.Pro527fs & 12 & $1(F)$ & No & No \\
\hline $\begin{array}{l}\text { Truncation occurring between } \\
\text { aa } 172 \text { and } 781\end{array}$ & c. $1648 C>T$ & p.Arg550* & 12 & $4(F)$ & Yes & Yes (1 child [4]) \\
\hline $\begin{array}{l}\text { Truncation occurring between } \\
\text { aa } 172 \text { and } 781\end{array}$ & c.1671dupA & p.Arg558Thrfs*9 & 12 & $1(F)$ & Yes & No \\
\hline $\begin{array}{l}\text { Truncation occurring between } \\
\text { aa } 172 \text { and } 781\end{array}$ & c. $1675 C>T$ & p.Arg559* & 12 & $1(\mathrm{~F})$ & Yes & Yes [4] \\
\hline $\begin{array}{l}\text { Truncation occurring between } \\
\text { aa } 172 \text { and } 781\end{array}$ & c. $1782 T>G$ & p.Tyr594* & 12 & $1(\mathrm{~F})$ & Yes & Yes [4] \\
\hline $\begin{array}{l}\text { Truncation occurring between } \\
\text { aa } 172 \text { and } 781\end{array}$ & c.1791delC & p.Tyr598Thrfs*18 & 12 & $1(F)$ & Yes & Yes [4] \\
\hline $\begin{array}{l}\text { Truncation occurring between } \\
\text { aa } 172 \text { and } 781\end{array}$ & c. $2038 \mathrm{~A}>\mathrm{T}$ & p.Lys $680^{*}$ & 13 & $1(\mathrm{~F})$ & Yes & Yes [4] \\
\hline $\begin{array}{l}\text { Truncation occurring between } \\
\text { aa } 172 \text { and } 781\end{array}$ & c.2047-1G > A & p.Gly683Valfs*101 & 13 & $1(\mathrm{~F})$ & Yes & Yes [4] \\
\hline $\begin{array}{l}\text { Truncation occurring between } \\
\text { aa } 172 \text { and } 781\end{array}$ & c. $2047-2 A>G$ & p.Gly683Cysfs*66 & 13 & $1(\mathrm{~F})$ & Yes & Yes [4] \\
\hline $\begin{array}{l}\text { Truncation occurring between } \\
\text { aa } 172 \text { and } 781\end{array}$ & c.2072_2073delCT & p.Ser691* & 14 & $1(\mathrm{~F})$ & Yes & Yes [4] \\
\hline $\begin{array}{l}\text { Truncation occurring between } \\
\text { aa } 172 \text { and } 781\end{array}$ & c.2258_2259delAA & p.Gln753Profs*10 & 15 & $1(\mathrm{~F})$ & No & No \\
\hline $\begin{array}{l}\text { Truncation occurring between } \\
\text { aa } 172 \text { and } 781\end{array}$ & $c .2376+5 G>A$ & p.(Lys760Tyrfs*10) & 16 & $1(\mathrm{~F})$ & Yes & Yes [4] \\
\hline Late truncation after aa 781 & c.2374dupA & p.Thr792Asnfs*9 & 16 & $1(F)$ & Yes & Yes [4] \\
\hline Late truncation after aa 781 & c.2377-8 T>A & p.(Val793Leufs*2) & 16 & $1(F)$ & No & No \\
\hline Late truncation after aa 781 & c. $2413 C>T$ & p.G $\ln 805^{*}$ & 17 & $1(\mathrm{M})$ & No & No \\
\hline Late truncation after aa 781 & c.2420_2430del & p.Ser807Cysfs*2 & 17 & $1(\mathrm{M})$ & No & No \\
\hline Late truncation after aa 781 & c.2504delC & p.Pro $835 \mathrm{Hisfs}^{*} 2$ & 17 & $1(\mathrm{~F})$ & Yes & Yes [4] \\
\hline Late truncation after aa 781 & c.2477-?_2713+?del & p.(Ser833Thrfs*22) & 17 & $2(F)$ & No & No \\
\hline Late truncation after aa 781 & c. $2564 C>G$ & p.Ser855* & 18 & $2(F)$ & Yes & Yes [4] \\
\hline Late truncation after aa 781 & c.2572delC & p.Arg858Alafs*5 & 18 & $1(F)$ & Yes & Yes [4] \\
\hline Late truncation after aa 781 & c.2635_2636delCT & p.Leu879Glufs*30 & 18 & $2(F)$ & Yes & Yes [4] \\
\hline Late truncation after aa 781 & c.2711delC & p.Pro904Glnfs*23 & 18 & $1(F)$ & Yes & Yes [4] \\
\hline \multicolumn{7}{|l|}{ Mutations not grouped } \\
\hline & c. $1612 A>G$ & p.Thr538Ala & 1 & $1(\mathrm{M})$ & No & No \\
\hline & c. $2684 C>T$ & p.Pro895Leu & 18 & $1(\mathrm{~F})$ & No & No \\
\hline & c.65-?_1944+?dup & p.(?) & $3-12$ & $1(\mathrm{M})$ & No & No \\
\hline & c.146-?_463+?dup & p.(?) & $5-7$ & $1(F)$ & No & No \\
\hline
\end{tabular}


Table 1 Distribution of CDKL5 mutations within our study group (Continued)

$\begin{array}{llllll}\text { (Position uncertain) } & \text { p.(?) } & 1-3 & 1(\mathrm{M}) & \text { Yes } & \text { Yes [4] } \\ \text { c.745-2A > G } & \text { p.(Phe249_Lys275del) } & 10 & 1(\mathrm{~F}) & \text { Yes } & \text { Yes [4] } \\ \text { c.825+1G > A } & \text { p.(Phe249_Lys275del) } & 10 & 1(\mathrm{~F}) & \text { Yes } & \text { Yes [4] } \\ \text { c.825+1G > T } & \text { p.(Phe249_Lys275del) } & 10 & 1(\mathrm{~F}) & \text { Yes } & \text { No } \\ \text { c.1030_1031insGAC } & \text { p.Lys344delinsArgGln } & 12 & 1(\mathrm{~F}) & \text { Yes } & \text { Yes [4] } \\ \text { c.2276+1G > A } & \text { p.(Val718_Trp759delinsGly) } & 15 & 1(\mathrm{~F}) & \text { Yes } & \text { Yes [4] }\end{array}$

CDKL5 numbering based on GenBank reference sequences NG_008475.1 and NM_003159.2, with the first A in the start codon numbered +1. Protein names in brackets indicate inferred changes based on in silico analyses, including splicing predictions by SpliceSiteFinder-like (accessed through Alamut Visual v2.3.4), MaxEntScan [22], NNSPLICE by the Berkeley Drosophila Genome Project [23], GeneSplicer [24] and Human Splicing Finder [25].

[3,7,31]. In the study by Bahi-Buisson and colleagues, only $1 / 20$ females was able to walk independently, a quarter had reported functional hand use and a quarter could use babble or single words [3]. The increased number of participants in our study compared to the French study is one likely explanation for the differing results. However, age may also be a factor as the median age of the females in the French study ( 4.5 years) was 2 years less than in ours. We have shown that there is a delay in the age of acquiring these developmental milestones and therefore

Table 2 Pathogenicity of missense mutations within our current sample of individuals with the CDKL5 disorder

\begin{tabular}{|c|c|c|c|c|c|}
\hline $\begin{array}{l}\text { Nucleotide } \\
\text { change (cDNA) }\end{array}$ & Protein change & SIFT (score 1-0) & MutationTaster ( $p$ value $0-1$ ) & PolyPhen2 (score 0-1) & Align GVGD (Class C0-C65) \\
\hline $\mathrm{c.38 \textrm {T }}>\mathrm{C}$ & p.Phe13Ser & Deleterious (0) & Disease-causing (0.999) & Probably damaging (0.999) & Benign (CO) \\
\hline C. $58 \mathrm{G}>\mathrm{C}$ & p.Gly20Arg & Deleterious (0) & Disease-causing (1) & Probably damaging (0.985) & Benign $(\mathrm{CO})$ \\
\hline C. $119 \mathrm{C}>\mathrm{T}$ & p.Ala40Val & Tolerated (0.12) & Disease-causing (0.999) & Probably damaging (0.999) & Benign (CO) \\
\hline C.191 T>C & p.Leu64Pro & Deleterious (0) & Disease-causing (1) & Probably damaging (1) & Benign (CO) \\
\hline c.364G > A & p.Ala122Thr & Deleterious (0) & Disease-causing (1) & Probably damaging (0.999) & Likely pathogenic (C55) \\
\hline c. $377 \mathrm{G}>\mathrm{A}$ & p.Cys126Tyr & Deleterious (0) & Disease-causing (1) & Probably damaging (1) & Pathogenic (C65) \\
\hline c.395 T> G & p.Val132Gly & Deleterious (0) & Disease-causing (1) & Probably damaging (1) & Pathogenic (C65) \\
\hline c. $428 \mathrm{~T}>\mathrm{A}$ & p. Ile143Asn & Deleterious (0) & Disease-causing (1) & Possibly damaging (0.9) & Benign (CO) \\
\hline c. $458 \mathrm{~A}>\mathrm{T}$ & p. Asp153Val & Deleterious (0) & Disease-causing (1) & Benign (0.07) & Benign (CO) \\
\hline C. $473 G>C$ & p.Arg158Pro & Deleterious (0) & Disease-causing (1) & Probably damaging (0.999) & Benign (CO) \\
\hline c. $514 \mathrm{G}>\mathrm{A}$ & p.Val172le & Deleterious (0) & Disease-causing (1) & Probably damaging (0.995) & Benign (CO) \\
\hline C. $526 \mathrm{~T}>\mathrm{C}$ & p. Trp176Arg & Deleterious (0) & Disease-causing (1) & Probably damaging (0.999) & Benign (CO) \\
\hline c. $532 \mathrm{C}>\mathrm{T}$ & p.Arg178Trp & Deleterious (0) & Disease-causing (1) & Probably damaging (1) & Benign (CO) \\
\hline c. $536 \mathrm{C}>\mathrm{T}$ & p. Ser179Phe & Deleterious (0) & Disease-causing (1) & Probably damaging (0.999) & Likely benign (C15) \\
\hline$c .577 G>C$ & p.Asp193His & Deleterious (0) & Disease-causing (1) & Probably damaging (1) & Pathogenic (C65) \\
\hline c. $587 \mathrm{C}>\mathrm{T}$ & p.Ser196Leu & Deleterious (0) & Disease-causing (1) & Probably damaging (0.976) & Pathogenic (C65) \\
\hline C.595 T > C & p.Cys199Arg & Deleterious (0) & Disease-causing (1) & Probably damaging (0.999) & Pathogenic (C65) \\
\hline c. $620 G>A$ & p.Gly207Glu & Deleterious (0) & Disease-causing (1) & Probably damaging (1) & Pathogenic (C65) \\
\hline c. $656 \mathrm{~A}>\mathrm{C}$ & p.Gln219Pro & Deleterious (0) & Disease-causing (1) & Probably damaging (0.996) & Pathogenic (C65) \\
\hline $\mathrm{c} .680 \mathrm{~T}>\mathrm{C}$ & p.Leu227Pro & Deleterious (0) & Disease-causing (1) & Probably damaging (0.996) & Pathogenic (C65) \\
\hline c. $2684 C>T$ & p.Pro895Leu & Deleterious (0.03) & Disease causing ( 1 ) & Possibly damaging (0.578) & Benign (CO) \\
\hline c. $872 \mathrm{G}>\mathrm{A}$ & p.Cys291Tyr & Deleterious (0.01) & Disease causing (1) & Probably damaging (0.930) & Benign (CO) \\
\hline C. $215 \mathrm{~T}>\mathrm{C}$ & p.lle72Thr & Deleterious (0) & Disease-causing (1) & Possibly damaging (0.578) & Benign (CO) \\
\hline c. $1612 A>G$ & p.Thr538Ala & Deleterious (0.04) & Polymorphism (1) & Benign (0.009) & Benign (CO) \\
\hline c.526 T > C & p.Trp167Arg & Deleterious (0) & Disease causing (1) & Probably damaging (0.999) & Benign (CO) \\
\hline
\end{tabular}

CDKL5 numbering based on GenBank reference sequences NG_008475.1 and NM_003159.2, with the first A in the start codon numbered +1. In silico predictions are carried out through Alamut Visual (v2.4) using SIFT (scale: $1=$ tolerated, $0=$ deleterious) [26], MutationTaster ( $p$ value for prediction confidence (not pathogenicity): $0=$ low confidence, $1=$ high confidence) [27], PolyPhen2 (HumVar module, scale: $0=$ benign, $1=$ probably damaging) [28], Align GVGD (scale: C0 = benign, C65 $=$ pathogenic) [29]. 


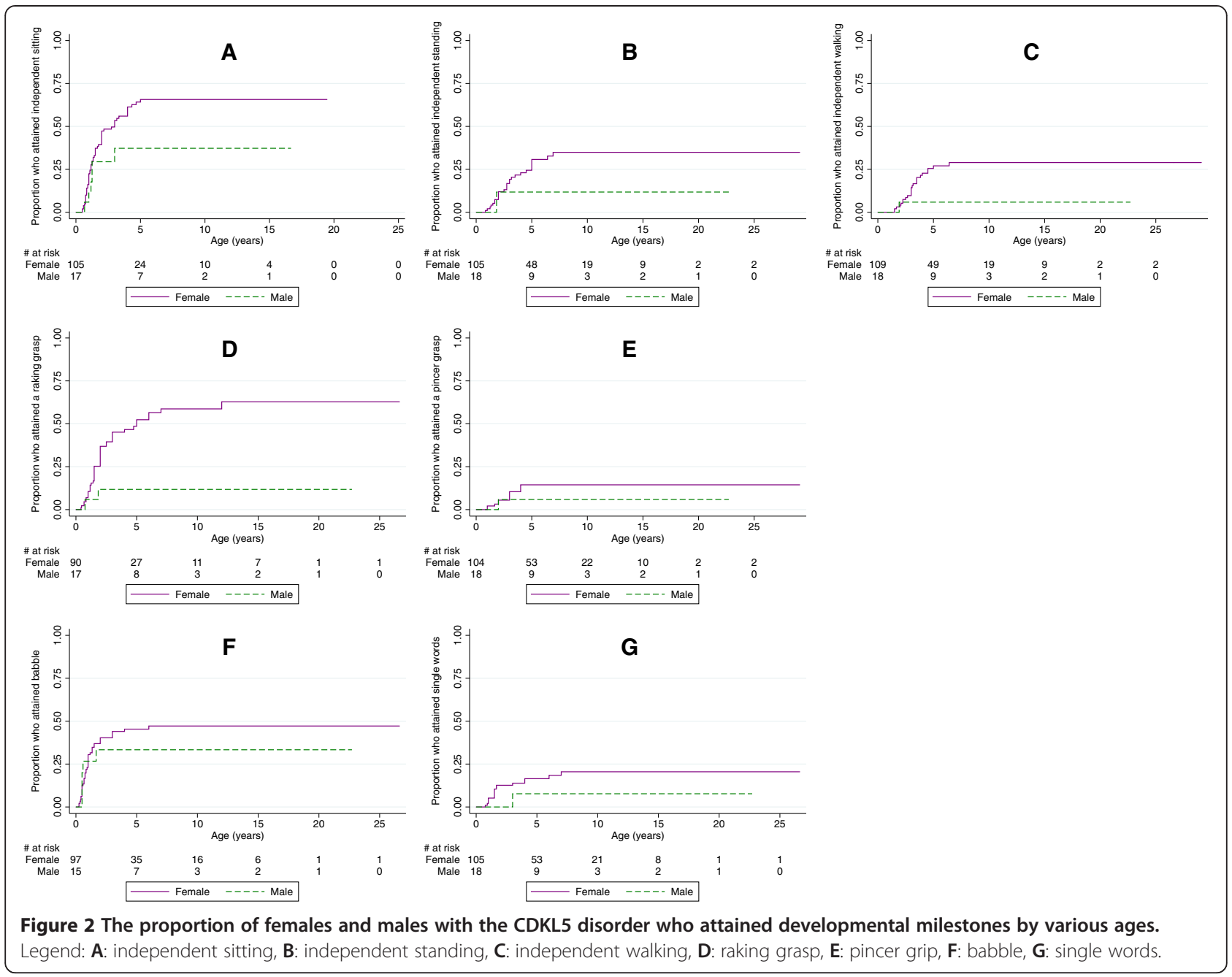

some of the girls in the French study may be yet to acquire these abilities. There also appears to be a 'cohort' effect in our data, with some of our older females (13 years and older) appearing to have attained less developmental milestones than those in the younger age group (7 to 13 years). In the past, children and adults with severe undiagnosed epilepsy disorders were targeted for screening for mutations in the CDKL5 gene. With advances in genetic technologies, less severely affected children are being tested and identified, thus increasing the variability seen initially in children with the CDKL5 disorder. Regardless, it is important that this variability in functional ability be recognised both by clinicians and researchers.

In our study, the males with the CDKL5 disorder had more impaired development than the females. Again, there was variability, with some of the males having less impaired development. Half of the boys in our study had attained rolling, a quarter learned to sit independently by 1 year 3 months and a quarter used babble by 7 months (earlier than the females in our group). There were four boys in our study who had attained more developmental milestones. The first was a 10-month old who could sit independently, babble and transfer objects from hand to hand $\left(\mathrm{c} .400 \mathrm{C}>\mathrm{T}, \mathrm{p} . \operatorname{Arg} 134^{*}\right)$. The second was a 2 and a half year old who could stand independently, transfer objects from hand to hand and babbled (c.351 T > A, p.Tyr $\left.117^{*}\right)$. The third was a 3 and a half year old who could sit independently, had limited hand use and could use single words (c.1612A > G, p.Thr538Ala). The fourth was a 4 and a half year old who had never had epilepsy, could walk independently (even run) and had a pincer grip but had language impairment (babble only) (c.514G > A, p.Val172Ile). There have been few boys with the CDKL 5 disorder described in the literature [7,9,12,18,32-38]. A recent study by Mirzaa and colleagues reported eight males with the CDKL5 disorder whose ages ranged from 2 months to 14 years (median age 5 years) [36]. These boys were described as having profound developmental delay, with impaired or absent language and motor abilities. The authors concluded that boys with the CDKL5 disorder are severely affected and have minimal acquisition of developmental skills. In our study, around half of 
Table 3 The proportion of individuals with the CDKL5 disorder of a particular current age who had attained various gross motor, communication and hand milestones

\begin{tabular}{|c|c|c|c|c|c|c|c|c|}
\hline \multirow[b]{3}{*}{ Developmental skill } & \multicolumn{8}{|c|}{ Age at completion of questionnaire } \\
\hline & \multicolumn{2}{|c|}{1.5 years and under } & \multicolumn{2}{|c|}{$1.5-7$ years } & \multicolumn{2}{|c|}{$7-13$ years } & \multicolumn{2}{|c|}{13 years and over } \\
\hline & $\begin{array}{l}\text { Females } \\
(n=11)\end{array}$ & $\begin{array}{l}\text { Males } \\
(n=1)\end{array}$ & $\begin{array}{l}\text { Females } \\
(n=50)\end{array}$ & $\begin{array}{l}\text { Males } \\
(n=10)\end{array}$ & $\begin{array}{l}\text { Females } \\
(n=32)\end{array}$ & $\begin{array}{l}\text { Males } \\
(n=5)\end{array}$ & $\begin{array}{l}\text { Females } \\
(n=16)\end{array}$ & $\begin{array}{l}\text { Males } \\
(n=2)\end{array}$ \\
\hline \multicolumn{9}{|l|}{ Gross motor } \\
\hline Roll front to back & $4(36.4)$ & $1(100)$ & $46(92)$ & $5(50)$ & $27(84)$ & $2(40)$ & $14(88)$ & $1(50)$ \\
\hline Roll back to front & $5(45)$ & $1(100)$ & $47(94)$ & $5(50)$ & $26(81)$ & $2(40)$ & $14(88)$ & $1(50)$ \\
\hline Transition from sitting to crawling position & $1(9)$ & $1(100)$ & $23(46)$ & $3(30)$ & $16(50)$ & $0(0)$ & $5(31)$ & $1(50)$ \\
\hline Crawl & $1(9)$ & $0(0)$ & $12(24)$ & $2(20)$ & $10(31)$ & $0(0)$ & $4(25)$ & $1(50)$ \\
\hline Stand with support & $2(18)$ & $1(100)$ & $37(74)$ & $3(30)$ & $22(69)$ & $1(20)$ & $10(63)$ & $1(50)$ \\
\hline Pull to stand & $1(9)$ & $0(0)$ & $13(26)$ & $2(20)$ & $14(44)$ & $1(20)$ & $6(38)$ & $1(50)$ \\
\hline Walk with support & $1(9)$ & $0(0)$ & $24(48)$ & $1(10)$ & $16(50)$ & $1(20)$ & $8(50)$ & $1(50)$ \\
\hline \multicolumn{9}{|l|}{ Fine motor } \\
\hline Transfer from hand to hand & $3(27)$ & $1(100)$ & $25(50)$ & $2(20)$ & $18(56)$ & $0(0)$ & $8(50)$ & $0(0)$ \\
\hline Play with blocks & $0(0)$ & $0(0)$ & $8(16)$ & $1(10)$ & $8(25)$ & $0(0)$ & $2(13)$ & $0(0)$ \\
\hline \multicolumn{9}{|l|}{ Social and communication } \\
\hline Social smile & $8(73)$ & $1(100)$ & $38(76)$ & $7(70)$ & $18(56)$ & $1(20)$ & $9(56)$ & $0(0)^{a}$ \\
\hline Fix and follow & $3(30.0)^{a}$ & $1(100)$ & $39(78)$ & $4(40)$ & $23(72)$ & $1(20)$ & $10(67)^{a}$ & $1(100)^{a}$ \\
\hline Wave goodbye & $0(0.0)$ & $0(0)$ & $11(22)$ & $1(10)$ & $4(13)$ & $0(0)$ & $1(6)$ & $0(0)$ \\
\hline Respond to own name & $4(36)$ & $1(100)$ & $33(66)$ & $3(33.3)^{a}$ & $20(63)$ & $2(40)$ & $9(56)$ & $0(0)$ \\
\hline Point to things that he/she wants & $0(0.0)$ & $0(0)$ & $6(12)$ & $1(10)$ & $8(26)^{a}$ & $0(0)$ & $2(13)$ & $0(0)$ \\
\hline Respond to others emotions & $4(36)$ & $1(100)$ & $23(47)^{a}$ & $4(40)$ & $11(34)$ & $1(20)$ & $4(25)$ & $1(50)$ \\
\hline Respond to 'no' & $2(18)$ & $0(0)$ & $16(32)$ & $2(25)^{a}$ & $13(41)$ & $0(0)$ & $4(25)$ & $0(0)$ \\
\hline Phrases & $0(0.0)$ & $0(0)$ & $3(6)$ & $0(0)$ & $3(9)$ & $0(0)$ & $1(6)$ & $0(0)$ \\
\hline
\end{tabular}

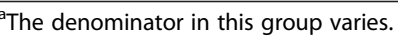

the males also had minimal acquisitions of developmental skills; however, the other half did attain some milestones. These findings show the variability in the acquisition of developmental milestones in males with the CDKL5 disorder. Although the males are generally more severe than females, more males achieved developmental milestones in our present study than previously reported. Our sample of boys was double that of the previously reported study, and so again increased sample size may be a key factor in being able to demonstrate this variability. Age could also be a factor, however the median ages of the boys in our and the US study [36] were similar (4.3 years and 5 years).

Table 4 The relationship between genotype and developmental milestone acquisition in females

\begin{tabular}{|c|c|c|c|c|c|c|c|c|}
\hline \multirow[t]{2}{*}{ Milestone } & \multicolumn{2}{|c|}{ No functional protein } & \multicolumn{2}{|c|}{$\begin{array}{l}\text { Missense/in-frame mutation } \\
\text { within catalytic domain }\end{array}$} & \multicolumn{2}{|c|}{$\begin{array}{l}\text { Truncation between aa } 172 \\
\text { and aa } 781 \text { inclusive }\end{array}$} & \multicolumn{2}{|c|}{ Truncation after aa 781} \\
\hline & Number (\%) & $\begin{array}{l}\text { Median age skill } \\
\text { attained (years) }\end{array}$ & Number (\%) & $\begin{array}{l}\text { Median age skill } \\
\text { attained (years) }\end{array}$ & Number (\%) & $\begin{array}{l}\text { Median age skill } \\
\text { attained (years) }\end{array}$ & Number (\%) & $\begin{array}{l}\text { Median age skill } \\
\text { attained (years) }\end{array}$ \\
\hline Sitting & 17/33 (52) & 2 & $18 / 27(67)$ & 3 & $10 / 26(38)$ & - & 10/12 (83) & 1.2 \\
\hline Standing & $6 / 32(19)$ & - & $5 / 28(18)$ & - & $8 / 28(29)$ & - & $5 / 10(50)$ & 5 \\
\hline Walking & 6/34 (18) & - & $4 / 28(14)$ & - & $6 / 28(21)$ & - & $5 / 12(42)$ & \\
\hline Babble & $13 / 29(45)$ & - & $13 / 27(48)$ & 6 & $12 / 25(44)$ & - & $4 / 8(50)$ & 1 \\
\hline Single words & $4 / 32(13)$ & - & $3 / 28(11)$ & - & $6 / 28(21)$ & - & $3 / 10(30)$ & \\
\hline Raking grasp & $12 / 29(44)$ & 7 & $16 / 25(64)$ & 4 & $8 / 22(36)$ & 12 & 7/9 (78) & 2 \\
\hline Pincer grasp & 3/32 (9) & - & $2 / 28(7)$ & - & $2 / 28(7)$ & - & $4 / 10(40)$ & - \\
\hline
\end{tabular}

(-) Values blank as less than $50 \%$ attained the particular skill. 


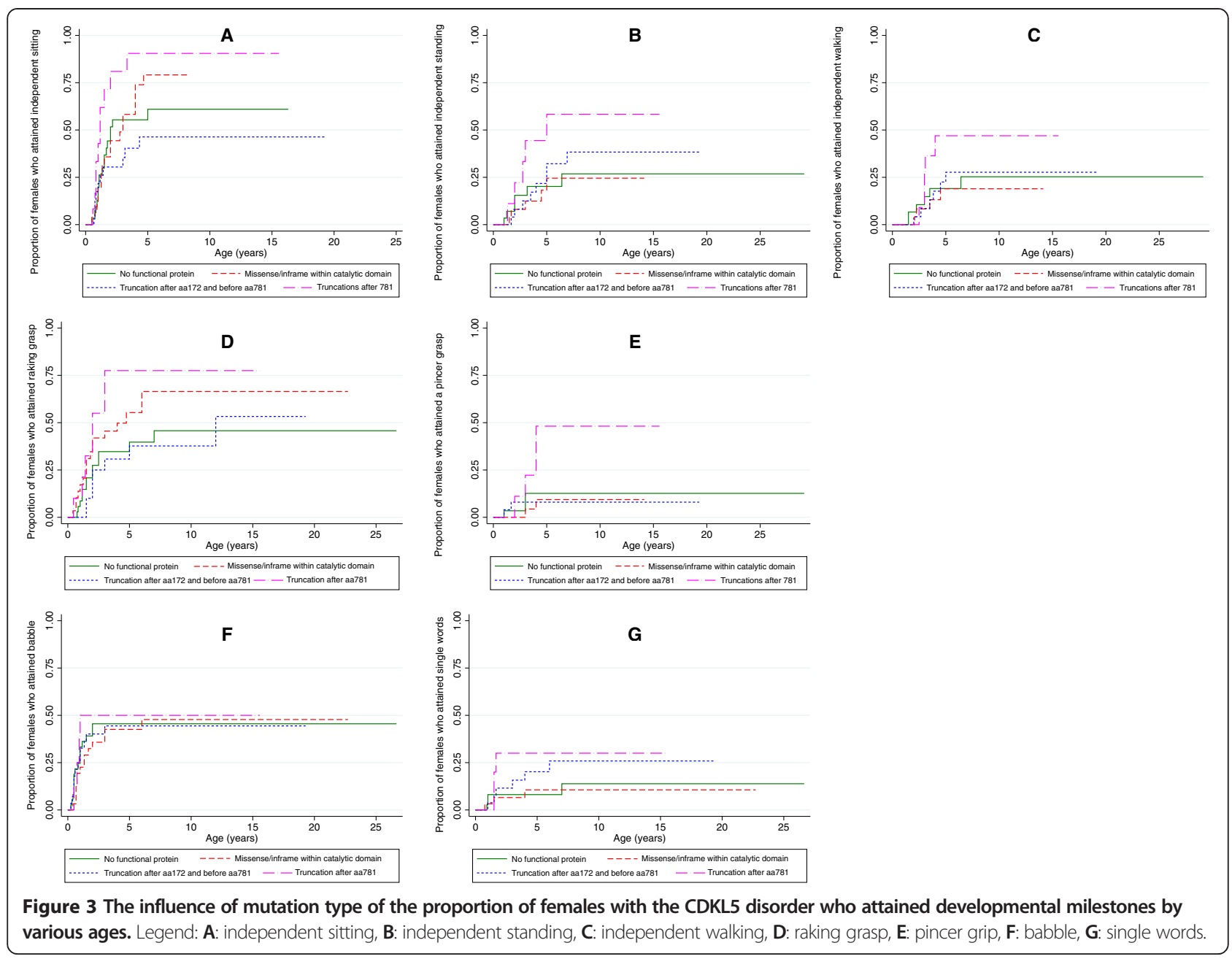

It is likely that the previously described 'cohort effect' may also be impacting on the variability seen in our male sample. The majority of males in the literature were identified through screening groups of severely affected individuals for CDKL5 mutations. Advances in genetic technology for diagnostic purposes mean those children who would previously never have been identified, such as our young boy who could run and who had never been diagnosed with epilepsy, are being diagnosed. These findings suggest that a different spectrum of boys with the CDKL5 disorder, who until now have not been diagnosed, may exist.

Our understanding of the functional role of CDKL5 is still limited, but CDKL5 has been shown to be involved in neuronal cell differentiation and proliferation, dendritic arborisation and dendritic spine morphology [39-41]. It is likely that mutations affecting the function of CDKL5 are likely to impair neuronal development and subsequent attainment of developmental milestones. The occurrence of early refractory epilepsy in the CDKL5 disorder may also contribute to the altered neuronal function, with seizures shown to impact developmental programming [42]. Whether the impairment seen in the CDKL5 disorder is a direct result of the occurrence of infantile seizures, resulting in an epileptic encephalopathy, or whether it is a consequence of the underlying gene mutation, is yet to be determined and further research in this area is needed.

The relationship between genotype and phenotype in the CDKL5 disorder has only been specifically investigated in one other study [43]. This study identified 12 patients (from a total of 26 with a mutation) who had one of eight recurrent mutations previously described in the literature. These 12 patients were pooled with an additional 14 individuals previously described in the literature. The authors found that those with the p.Ala40Val, which is a missense mutation within the ATP binding site of the kinase domain, had a milder phenotype (phenotype severity based on factors such as gross motor ability, hand function, stereotypies and seizure severity) than those with a missense mutation elsewhere in the kinase domain or a frameshift within the C-terminal region. Due to the heterogeneity of mutations within the CDKL5 disorder, we chose not to investigate specific mutations, rather, we 
pooled together different types that were predicted to have similar functional/structural consequences. This heterogeneity means that genotype/phenotype studies will be more difficult to conduct unless larger numbers of individuals are ascertained. We found no clear relationship between genotype and phenotype in our study, but there were a few differences between the groups. Females with a truncation between aa 172 and aa 781 attained fewer developmental milestones, similar to that of those who lacked the entire CDKL5 gene. Earlier research suggested that loss of the C-terminal region can in fact act as a loss of function mutation due to loss of localisation ability and increased self-phosphorylation (increased capacity to phosphorylate the kinase itself) [16,44]. One difference we found was that individuals with a truncating mutation after aa 781, such as the mutation c.2635_2636delCT, appeared to attain more milestones overall. This differs from the previous study, which found that girls with this mutation were more severely affected and unable to walk [43]. No clear conclusion can be drawn on the role of genotype until even larger studies are undertaken and other previously identified factors (such as epilepsy) are considered, although it does appear as though genotype may have a part to play in the clinical variability in the CDKL5 disorder.

Our study is the first major international data collection of individuals with the CDKL5 disorder. This gives us the advantage of having the largest data collection on individuals with the CDKL5 disorder. Our data collection tools were also designed to capture clinical information specific to the CDKL5 disorder, not other disorders such as RTT. It is apparent that there is much variability within this disorder, and therefore larger numbers are still needed to provide a more comprehensive representation of the clinical presentation. Recall error, especially for caregivers with older children, is likely. We have limited this by asking caregivers to refer to their child's medical records and diaries before completing the questionnaire. Because these children exhibit symptoms from an early age, developmental gains are most likely to be well recorded, as caregivers are paying special attention to their child's development. We found that many parents knew whether their child attained a particular milestone, but the age that these skills were attained was not always known, which is demonstrated by the missing data in our study. Changes in diagnostic technology and increased awareness of the CDKL5 disorder mean that children are being diagnosed younger, and a more variable spectrum is being identified. This results in the 'cohort effect' to which we have alluded, where the younger are less severe than previously diagnosed children. The identification of younger children means that this data collection will have the capacity to collect prospective data on developmental milestones. Finally, our study will only reach caregivers of individuals who have access to the internet and therefore selection bias exists in that regard.

\section{Conclusion}

Understanding the acquisition of gross motor milestones in the CDKL5 disorder is valuable information for caregivers, their therapists and clinicians and shows that for most, skills are attained at a much later age. It would be of interest to further investigate the events surrounding the acquisition of these milestones, especially with regard to seizure control. For caregivers, our data provide hope that their young child may still attain milestones at a later age than is usual for the general population. As the database grows, it will be important, to further examine the relationship between genotype and functional abilities. Individuals with the CDKL5 disorder have previously been described as having severely impaired development. Our findings show the variability within this disorder and suggest that there may be children with the CDKL5 disorder who present differently to the clinical picture originally described. Although we found no clear relationship between genotype and phenotype, the differences between individuals suggest that with greater numbers such a relationship may be identified. Continued research into the natural history of the CDKL5 disorder is needed to further our understanding of the variability seen within this condition.

\section{Abbreviations}

CDKL5: cyclin-dependent kinase-like 5; InterRett: International Rett syndrome Phenotype Database; RTT: Rett syndrome; Aa: amino acid.

\section{Competing interests}

The authors declare that they have no competing interests.

\section{Authors' contributions}

SF conceptualised and designed the study, designed the data collection tools, carried out the analysis, drafted the initial manuscript and revised manuscript; HL and JD supervised the study and data collection design, assisted in data collection, and reviewed and revised all manuscript draughts; ND contributed to study design and data analysis and reviewed the initial manuscript; JC contributed to the study design and analysis of mutation data and reviewed and revised the initial manuscript; GH provided detailed input into the categorisation of mutation data, including classification of pathogenicity, and reviewed and revised the initial manuscript; SW advised on some aspects of the study design and reviewed and revised the initial manuscript; DF provided supervision to the first author and reviewed and revised the initial manuscript. All authors have approved the submitted manuscript.

\section{Acknowledgements}

The authors would like to express their special appreciation to all the families who have participated in the International CDKL5 Disorder Database. We would like to acknowledge the International Foundation for CDKL5 Research for their ongoing support and assistance and Dr. Alison Anderson for her assistance in database design and establishment. This study is supported by Ms. Fehr's Australian Postgraduate Award, University of Western Australia Safety-Net Top-Up Scholarship, Stan and Jean Perron Top-Up Scholarship and Stan and Jean Perron Award for Excellence. Data collection was also supported by an NHMRC Program Grant No.572742 (Dr Jenny Downs) and NHMRC Senior Research Fellowship No. 572568 (Dr. Helen Leonard). 


\section{Author details}

${ }^{1}$ Telethon Kids Institute, University of Western Australia, Perth, Western Australia, Australia. ${ }^{2}$ School of Physiotherapy and Exercise Science, Curtin University, Perth, Western Australia, Australia. ${ }^{3}$ Western Sydney Genetics Program, Children's Hospital at Westmead, Sydney, NSW, Australia. ${ }^{4}$ Disciplines of Paediatrics \& Child Health and Genetic Medicine, University of Sydney, Sydney, NSW, Australia. ${ }^{5}$ Department of Neurology and Rehabilitation, Princess Margaret Hospital, Perth, Western Australia, Australia. ${ }^{6}$ School of Paediatrics and Child Health, University of Western Australia, Perth, Western Australia, Australia.

\section{Received: 7 October 2014 Accepted: 16 December 2014} Published: 5 January 2015

\section{References}

1. Jähn J, Caliebe A, von Spiczak S, Boor R, Stefanova I, Stephani U, et al. CDKL5 mutations as a cause of severe epilepsy in infancy: clinical and electroencephalographic long-term course in 4 patients. J Child Neurol. 2013;28:937-41.

2. Stalpers $X L$, Spruijt $L$, Yntema HG, Verrips A. Clinical phenotype of 5 females with a CDKL5 mutation. J Child Neurol. 2012;27:90-3.

3. Bahi-Buisson N, Nectoux J, Rosas-Vargas H, Milh M, Boddaert N, Girard B, et al. Key clinical features to identify girls with CDKL5 mutations. Brain. 2008;131:2647-61.

4. Fehr S, Wilson M, Downs J, Williams S, Murgia A, Sartori S, et al. The CDKL5 disorder is an independent clinical entity associated with early-onset encephalopathy. Eur J Hum Genet. 2013;21:266-73.

5. Neul JL, Kaufmann WE, Glaze DG, Christodoulou J, Clarke AJ, Bahi-Buisson N, et al. Rett syndrome: revised diagnostic criteria and nomenclature. Ann Neurol. 2010:68:944-50.

6. Olson HE, Poduri A. CDKL5 mutations in early onset epilepsy: case report and review of the literature. J Pediatr Epilep. 2012;1:151-9.

7. Liang JS, Shimojima K, Takayama R, Natsume J, Shichiji M, Hirasawa K, et al. CDKL5 alterations lead to early epileptic encephalopathy in both genders. Epilepsia. 2011;52:1835-42.

8. White R, Ho G, Schmidt S, Scheffer IE, Fischer A, Yendle SC, et al. Cyclin-dependent kinase-like 5 (CDKL5) mutation screening in Rett syndrome and related disorders. Twin Res Hum Genet. 2010;13:168-78.

9. Bartnik M, Derwinska K, Gos M, Obersztyn E, Kolodziejska K, Erez A, et al. Early-onset seizures due to mosaic exonic deletions of CDKL5 in a male and two females. Genet Med. 2011:13:447-52.

10. Martínez AR, Armstrong J, Gerotina E, Fons C, Campistol J, Pineda M. CDKL5 in different atypical Rett syndrome variants: description of the first eight patients from Spain. J Pediatr Epilep. 2012;1:27-35.

11. Pini G, Bigoni S, Engerström IW, Calabrese O, Felloni B, Scusa MF, et al. Variant of Rett syndrome and CDKL5 gene: clinical and autonomic description of 10 cases. Neuropediatrics. 2013;43:37-43.

12. Weaving LS, Christodoulou J, Williamson SL, Friend KL, McKenzie OL, Archer $\mathrm{HL}$, et al. Mutations of CDKL5 cause a severe neurodevelopmental disorder with infantile spasms and mental retardation. Am J Hum Genet. 2004;75:1079-93.

13. Bebbington A, Anderson A, Ravine D, Fyfe S, Pineda M, de Klerk N, et al. Investigating genotype-phenotype relationships in Rett syndrome using an international dataset. Neurology. 2008;70:868-75.

14. Cuddapah VA, Pillai RB, Shekar KV, Lane JB, Motil KJ, Skinner SA, et al. Methyl-CpG-binding protein 2 (MECP2) mutation type is associated with disease severity in Rett syndrome. J Med Genet. 2014; doi:10.1136/jmedgenet2013-102113.

15. Hagberg B, Gillberg C. Rett Variants—rettoid phenotypes. In: Hagberg B, editor. Rett Syndrome-Clinical and Biological Aspects. London: Mac Keith Press; 1993.

16. Bertani I, Rusconi L, Bolognese F, Forlani G, Conca B, De Monte L, et al. Functional consequences of mutations in CDKL5, an X-linked gene involved in infantile spasms and mental retardation. J Biol Chem. 2006:281:32048-56.

17. Kilstrup-Nielsen C, Rusconi L, La Montanara P, Ciceri D, Bergo A, Bedogni F, et al. What we know and would like to know about CDKL5 and its involvement in epileptic encephalopathy. Neural Plast. 2012;2012:728267.

18. Castrén M, Gaily E, Tengström C, Lähdetie J, Archer H, Ala-Mello S. Epilepsy caused by CDKL5 mutations. Eur J Paediatr Neurol. 2011;15:65-9.
19. Archer HL, Evans J, Edwards S, Colley J, Newbury-Ecob R, O'Callaghan F, et al. CDKL5 mutations cause infantile spasms, early onset seizures, and severe mental retardation in female patients. J Med Genet. 2006:43:729-34

20. Rademacher N, Hambrock M, Fischer U, Moser B, Ceulemans B, Lieb W, et al Identification of a novel CDKL5 exon and pathogenic mutations in patients with severe mental retardation, early-onset seizures and Rett-like features. Neurogenetics. 2011;12:165-7.

21. Fichou Y, Bieth E, Bahi-Buisson N, Nectoux J, Girard B, Chelly J, et al. Re: CDKL5 mutations in boys with severe encephalopathy and early-onset intractable epilepsy. Neurology. 2009;73:77-8. author reply 78.

22. Yeo G, Burge CB. Maximum entropy modeling of short sequence motifs with applications to RNA splicing signals. J Comput Biol. 2004;11:377-94.

23. Reese MG, Eeckman FH, Kulp D, Haussler D. Improved splice site detection in Genie. J Comput Biol. 1997:4:311-23.

24. Pertea M, Lin X, Salzberg SL. GeneSplicer: a new computational method for splice site prediction. Nucleic Acids Res. 2001;29:1185-90.

25. Desmet F-O, Hamroun D, Lalande M, Collod-Béroud G, Claustres M, Béroud C. Human Splicing Finder: an online bioinformatics tool to predict splicing signals. Nucleic Acids Res. 2009;37:e67.

26. Ng PC, Henikoff S. Predicting deleterious amino acid substitutions. Genome Res. 2001;11:863-74.

27. Schwarz JM, Rodelsperger C, Schuelke M, Seelow D. MutationTaster evaluates disease-causing potential of sequence alterations. Nat Methods. 2010;7:575-6.

28. Adzhubei IA, Schmidt S, Peshkin L, Ramensky VE, Gerasimova A, Bork P, et al. A method and server for predicting damaging missense mutations. Nat Methods. 2010:7:248-9.

29. Tavtigian SV, Deffenbaugh AM, Yin L, Judkins T, Scholl T, Samollow PB, et al. Comprehensive statistical study of 452 BRCA1 missense substitutions with classification of eight recurrent substitutions as neutral. J Med Genet. 2006:43:295-305

30. WHO multicentre growth reference study group. WHO Motor Development Study: Windows of achievement for six gross motor development milestones. Acta Paediatr. 2006;95:86-95.

31. Artuso R, Mencarelli MA, Polli R, Sartori S, Ariani F, Pollazzon M, et al. Early-onset seizure variant of Rett syndrome: definition of the clinical diagnostic criteria. Brain Dev. 2010;32:17-24.

32. Elia M, Falco M, Ferri R, Spalletta A, Bottitta M, Calabrese $G$, et al. CDKL5 mutations in boys with severe encephalopathy and early-onset intractable epilepsy. Neurology. 2008;71:997-9.

33. Jansen A, Bauters M, De Rademaeker M, De Meirleir L, Froyen G, Fryns JP, et al. Epileptic encephalopathy in a boy with an interstitial deletion of Xp22 comprising the CDKL5 gene. Epilepsia. 2006;47:367.

34. Masliah-Plachon J, Auvin S, Nectoux J, Fichou Y, Chelly J, Bienvenu T. Somatic mosaicism for a CDKL5 mutation as an epileptic encephalopathy in males. Am J Med Genet. 2010;152A:2110-1. doi:10.1002/ajmg.a.33037.

35. Melani F, Mei D, Pisano T, Savasta S, Franzoni E, Ferrari A, et al. CDKL5 gene-related epileptic encephalopathy: electroclinical findings in the first year of life. Dev Med Child Neurol. 2011;53:354-60.

36. Mirzaa GM, Paciorkowski AR, Marsh ED, Berry-Kravis EM, Medne L, Alkhateeb A, et al. CDKL5 and ARX mutations in males with early-onset epilepsy. Pediatr Neurol. 2013;48:367-77.

37. Nishimura A, Takano T, Mizuguchi T, Saitsu H, Takeuchi $Y$, Matsumoto N. CDKL5 disruption by $\mathrm{t}(\mathrm{X} ; 18)$ in a girl with West syndrome. Clin Genet. 2008;74:288-90.

38. Sartori S, Di Rosa G, Polli R, Bettella E, Tricomi G, Tortorella G, et al. A novel CDKL5 mutation in a 47, XXY boy with the early-onset seizure variant of Rett syndrome. Am J Med Genet. 2009;149A:232-6.

39. Chen QA, Zhu YC, Yu J, Miao S, Zheng J, Xu L, et al. CDKL5, a protein associated with Rett syndrome, regulates neuronal morphogenesis via Rac1 signaling. J Neurosci. 2010;30:12777-86.

40. Ricciardi S, Ungaro F, Hambrock M, Rademacher N, Stefanelli G, Brambilla D, et al. CDKL5 ensures excitatory synapse stability by reinforcing NGL-1-PSD95 interaction in the postsynaptic compartment and is impaired in patient iPSC-derived neurons. Nat Cell Biol. 2012;14:911-23.

41. Valli E, Trazzi S, Fuchs C, Erriquez D, Bartesaghi R, Perini G, et al. CDKL5, a novel MYCN-repressed gene, blocks cell cycle and promotes differentiation of neuronal cells. Biochim Biophys Acta. 2012;1819:1173-85.

42. Nardou R, Ferrari DC, Ben-Ari Y. Mechanisms and effects of seizures in the immature brain. Semin Fetal Neonatal Med. 2013;18:175-84. 
43. Bahi-Buisson N, Villeneuve N, Caietta E, Jacquette A, Maurey H, Matthijs G, et al. Recurrent mutations in the CDKL5 gene: genotype-phenotype relationships. Am J Med Genet A. 2012;158A:1612-9.

44. Rusconi L, Salvatoni L, Giudici L, Bertani I, Kilstrup-Nielsen C, Broccoli V, et al. CDKL5 expression is modulated during neuronal development and its subcellular distribution is tightly regulated by the C-terminal tail. J Biol Chem. 2008;283:30101-11.

doi:10.1186/1866-1955-7-2

Cite this article as: Fehr et al:: There is variability in the attainment of developmental milestones in the CDKL5 disorder. Journal of

Neurodevelopmental Disorders 2015 7:2.

\section{Submit your next manuscript to BioMed Central and take full advantage of:}

- Convenient online submission

- Thorough peer review

- No space constraints or color figure charges

- Immediate publication on acceptance

- Inclusion in PubMed, CAS, Scopus and Google Scholar

- Research which is freely available for redistribution 